\title{
Pseudosleep events in patients with psychogenic non- epileptic seizures: prevalence and associations
}

\author{
R Duncan, M Oto, A J C Russell, P Conway
}

J Neurol Neurosurg Psychiatry 2004;75:1009-1012. doi: 10.1136/jnnp.2003.022632

\begin{abstract}
See end of article for authors' affiliations

.....................

Correspondence to: Dr Roderick Duncan, West of Scotland Regional Epilepsy Service, Institute of Neurological Sciences, Southern General Hospital, Glasgow G51 4TF, UK; r.duncan@ clinmed.gla.ac.uk
\end{abstract}

Received 7 July 2003 Revised 17 October 2003 Accepted

14 November 2003

\begin{abstract}
Objectives: To determine the prevalence and clinical associations of a history of events during sleep in patients with psychogenic non-epileptic seizures (PNES, pseudoseizures), and to compare the prevalence of a history of sleep events with that in poorly controlled epilepsy.

Methods: Prospective study by semistructured interview of the history of event patterns and their clinical associations in 142 patients with video EEG confirmed PNES, and 100 patients with poorly controlled epilepsy.

Results: $84 / 142$ patients with PNES (59\%) and 47/100 with epilepsy (47\%) gave a history of events during sleep $(p=0.062)$. In patients with PNES, significant associations were found between a history of sleep events and: convulsive clinical semiology, antiepileptic drug treatment, fatigue, suicide attempts, mood disorder, and physical abuse. A particularly strong association with social security benefit was also found (odds ratio 4.0, $\mathrm{p}<0.001$ ).

Conclusions: The prevalence of a history of sleep events is similar in PNES and epilepsy, and is of no value in discriminating between the two, although a history of events occurring exclusively during sleep does suggest epileptic seizures. The clinical associations found indicate that a combination of psychopathological and external influences may be important in determining whether or not a patient with PNES gives a history of events during sleep.
\end{abstract}

$\mathrm{P}$ atients with psychogenic non-epileptic seizures (PNES pseudoseizures) comprise up to $24 \%$ of referrals to clinics for intractable epilepsy, ${ }^{1}$ and one population based study has suggested that they comprise $5 \%$ of patients thought to have epilepsy. ${ }^{2}$ Diagnostic delay remains common and exposes patients to the side effects of inappropriate anticonvulsant drugs, ${ }^{3}$ particularly where the events take a "convulsive" form. ${ }^{5}$ A century ago, Gowers stated that events arising from sleep were not psychogenic. ${ }^{6}$ This rule remains commonly held, and has been stated on various occasions since, $^{78}$ including recently. ${ }^{9}$ It has, however, been shown that PNES may arise during periods when the patient is apparently asleep but when electroencephalography (EEG) shows them to be awake (a situation termed "pseudosleep" ${ }^{\prime \prime}{ }^{10}{ }^{11}$ If pseudosleep PNES occur outside hospital (or indeed if patients falsely claim that events occur during sleep outside hospital), then clinicians might fail to challenge an erroneous diagnosis, or might make an erroneous diagnosis in the first place, based on the belief that sleep related seizures are diagnostic of epilepsy.

We have carried out a prospective study of a large patient group with video EEG confirmed PNES with the aims of determining what proportion of patients with PNES give a history of events during sleep, whether a history of events during sleep is associated with some indices of increased difficulty of diagnosis, and whether such patients have a distinct clinical profile. We have also measured the prevalence of a history of events during sleep in a comparator group of patients with poorly controlled epilepsy.

\section{METHODS}

We included in the study all 160 new patients seen at the Glasgow PNES clinic between January 2000 and March 2002 in whom video EEG confirmed the diagnosis of PNES. The diagnostic process included careful comparison of recorded events with eyewitness descriptions, and a review of video EEG recordings with witnesses in cases of doubt, to make sure that the recorded events corresponded with those that had been witnessed, and that all types of event had been recorded. Patients were included whether or not they had ictal impairment of consciousness. At the time of initial assessment, a range of clinical data relating to sleep events and their clinical associations was acquired by standardised semistructured interview of the patient and eyewitness. Eighteen patients who had evidence of past or present concomitant epilepsy were excluded from the study according to our standard criteria (past or present event descriptions suggesting epilepsy, onset of events in early childhood, epileptiform abnormalities in the interictal EEG, previous recording of epileptic seizures). This left 142 consecutive patients with PNES only; 103 (72.5\%) were female and 39 $(27.5 \%)$ male. Their age range was 13 to 72 years (mean (SD), $37.3(13.7))$.

A comparator group of 100 consecutive patients with poorly controlled epilepsy (more than four seizures a month) seen at an epilepsy clinic between March and June 2003 was also interviewed, using only those questions from the semistructured interview relating to the sleep/wake pattern of their seizures; 51 were male, 49 female, with an age range of 18 to 76 years (mean 39.40 (15.27)). The difference in sex ratio between epilepsy and PNES groups was significant $\left(\mathrm{p}=0.001, \chi^{2}\right)$.

In view of the potential diagnostic implications, patients in both groups were recorded as having sleep events even if such events were only occasional. Patients were recorded as giving a history of waking events only, events during sleep only, or both. No data were collected regarding the precise timing of events during sleep, as initial attempts to do so suggested the data would be incomplete in most patients.

For purposes of analysis, the proportion of patients in the PNES group giving a history of events during sleep was

Abbreviation: PNES, psychogenic non-epileptic seizure 
compared with the proportion of patients in the epilepsy group giving a history of events during sleep. The PNES patients were divided into two groups-those reporting sleep events at the time of clinical assessment and those not reporting sleep events. These two PNES groups were then compared, and associations between a history of events during sleep and a number of other variables were tested (table 1) using Fisher's exact test or a $\chi^{2}$ test where more than two categories were analysed (for example, the number of computed tomography (CT) scans was categorised as 0,1 , or more than 1 ). The difference between groups in time from onset of PNES to confirmed diagnosis was also tested as a continuous variable using the Mann-Whitney U test. As this was an exploratory study, and as the comparisons in table 1 were determined by previous hypothesis and were the only ones tested, correction for $\alpha$ error was not applied.

\section{RESULTS}

Screening of PNES data found 18 missing results, or $0.79 \%$ of entries. Ten of these were accounted for by two patients who had complied poorly with data acquisition. Additionally, in 16 patients it was not possible to determine the precise onset of PNES, so these were not included in analysis of diagnostic delay. Other than this, the maximum number of missing values was 3 in any category.

\section{Prevalence of history of sleep events in patients with PNES $v$ patients with epilepsy}

A history of sleep events was given by 84 of 142 PNES patients $(59.2 \%)$, and 47 of 100 patients with uncontrolled epilepsy $(47.0 \%)\left(p=0.062, \chi^{2}\right)$. In both groups, the proportion of sleep to wake events varied widely. One PNES patient had events occurring only during sleep, compared with 16 patients with uncontrolled epilepsy $\left(p<0.001, \chi^{2}\right)$. In all but two of the PNES patients, the history of events appearing to occur during sleep was confirmed by the eyewitness.
PNES patients: comparison of those with and without a history of sleep events

Table 1 shows the variables analysed, the significance of the difference between groups, the odds ratio (where analysis was by $2 \times 2$ table), and the $95 \%$ confidence intervals (CI). Two-tailed $\mathrm{p}$ values are quoted.

Patients with a history of events during sleep had a mean time from first event to diagnosis of 58.8 months, and without pseudosleep events, 54.5 months—a small difference that was not statistically significant. The number of tests carried out before the diagnosis was in general small. Overall, the number of EEGs ranged from 0 to 4, the number of sleep EEGs from 0 to 3, and the number of CT scans from 0 to 5 . There were no significant differences between groups. Patients with a history of events during sleep were 1.8 times more likely to be on antiepileptic drugs at the time of presentation to the PNES clinic, but this difference failed to reach statistical significance. However, patients with a history of events during sleep were 2.8 times more likely to be on more than one antiepileptic drug (95\% CI, 1.11 to 13.43 ; $\mathrm{p}=0.023)$.

Event frequency was banded and analysed as a categorical variable and showed no differences between the two groups. Convulsive semiology was, however, 2.8 times more likely in patients with a history of events during sleep (95\% CI, 1.34 to 5.97; $p=0.005)$, and patients with a history of events during sleep were 4.0 times more likely to be claiming social security benefits $(1.85$ to $8.73 ; \mathrm{p}<0.001)$. Medically unexplained symptoms in general were not significantly more common in patients with a history of events during sleep, with the exception of fatigue (odds ratio 2.1 (95\% CI, 1.46 to 4.21 ), $\mathrm{p}=0.032$ ), and there was an increase in parasuicide (odds ratio 2.6 ( 1.07 to 6.27$), p=0.023$ ) and mood disorder (in most cases depression) (odds ratio 2.2 (1.09 to 2.19), $\mathrm{p}=0.020)$.

There was no significant association between a history of events during sleep and sexual or physical abuse (in the case of physical abuse, the association just failed to reach significance), or with other traumatic experiences, although

Table 1 Patients with psychogenic non-epileptic seizures: comparisons between those with and without a history of events during sleep

\begin{tabular}{|c|c|c|c|c|c|}
\hline Hypothesis & Variable & $\begin{array}{l}\text { Degrees of freedom (df) } \\
\text { for } \chi^{2} \text { unless stated }\end{array}$ & p Value & $\begin{array}{l}\text { Odds } \\
\text { ratio }\end{array}$ & $95 \% \mathrm{Cl}$ \\
\hline \multirow{5}{*}{$\begin{array}{l}\text { Pseudosleep PNES delay diagnosis } \\
\text { or make diagnosis more difficult }\end{array}$} & \multirow{5}{*}{$\begin{array}{l}\text { Time from onset of PNES to diagnosis } \\
\text { Number of interictal and sleep EEGs } \\
\text { carried out prior to diagnosis } \\
\text { Number of CT scans carried out prior to } \\
\text { diagnosis } \\
\text { On antiepileptic drugs at time of presentation } \\
\text { On multiple antiepileptic drugs at time of } \\
\text { presentation }\end{array}$} & $M W U=1434.5 / \mathrm{df}=112$ & 0.495 & $n / a$ & $\mathrm{n} / \mathrm{a}$ \\
\hline & & $d f=3$ & 0.439 & $n / a$ & $\mathrm{n} / \mathrm{a}$ \\
\hline & & $d f=2$ & 0.266 & $\mathrm{n} / \mathrm{a}$ & $\mathrm{n} / \mathrm{a}$ \\
\hline & & $d f=1$ & 0.093 & 1.8 & 0.9 to 3.58 \\
\hline & & $d f=1$ & 0.033 & 2.8 & 1.11 to 13.43 \\
\hline \multirow{8}{*}{$\begin{array}{l}\text { Pseudosleep events are associated } \\
\text { with severe PNES disorder }\end{array}$} & Event frequency & $d f=6$ & 0.362 & $\mathrm{n} / \mathrm{a}$ & $\mathrm{n} / \mathrm{a}$ \\
\hline & Event semiology (convulsive $v$ other) & $d f=1$ & 0.006 & 2.8 & 1.34 to 5.97 \\
\hline & Social security benefits & $d f=1$ & $<0.001$ & 4.0 & 1.85 to 8.73 \\
\hline & Employment & $d f=1$ & 0.441 & 0.75 & 0.37 to 1.55 \\
\hline & Fatigue & $d f=1$ & 0.032 & 2.1 & 1.06 to 4.21 \\
\hline & Parasuicide & $d f=1$ & 0.030 & 2.6 & 1.07 to 6.27 \\
\hline & Medically unexplained symptoms & $d f=1$ & 0.271 & 1.6 & 0.69 to 3.80 \\
\hline & Mood disorder & $d f=1$ & 0.026 & 2.2 & 1.09 to 2.19 \\
\hline \multirow{3}{*}{$\begin{array}{l}\text { Pseudosleep PNES are associated } \\
\text { with sexual or physical abuse, or } \\
\text { other traumatic experience }\end{array}$} & Sexual abuse & & 0.103 & 1.81 & 0.88 to 3.68 \\
\hline & Physical abuse & $d f=1$ & 0.052 & 2.14 & 0.99 to 2.14 \\
\hline & Other traumatic experience & $d f=1$ & 0.485 & 1.31 & 0.62 to 1.31 \\
\hline $\begin{array}{l}\text { Pseudosleep PNES are associated } \\
\text { with sleep disturbance }\end{array}$ & Disturbed sleep pattern & $d f=1$ & 0.166 & 1.74 & 0.79 to 3.81 \\
\hline
\end{tabular}


the odds ratios did suggest a trend. There was no significant association between a history of events during sleep and sleep disturbance.

\section{DISCUSSION}

Studies of PNES have found that between $10 \%$ and $60 \%$ of patients also have epilepsy, the majority being at the lower end of this range. ${ }^{12}$ At $11.25 \%$, our figure was consistent with this. Video EEG usually allows confident distinction between epileptic seizures and PNES on the basis of typical clinical features and EEG changes. Occasionally, seizures may pass with little or no EEG change, or more commonly with the EEG obscured by muscle artefact. This may pose a problem in distinguishing PNES from certain uncommon types of frontal seizure. There are various clinical factors that can distinguish the two (notably duration), ${ }^{13}{ }^{14}$ and these factors were borne in mind during this study. Additionally, patients were excluded from the study if they had any evidence of epilepsy in the shape of interictal epileptic discharges or events during childhood.

In series of patients admitted for video EEG, Benbadis et al recorded pseudosleep PNES in 10 of 18 patients, or $55 \% .^{10}$ Thacker et al found 12 of 103 patients with pseudosleep PNES, or $11.5 \%{ }^{11}$ Benbadis' population was relatively small, so comparison with our series may not be valid. Our figure was much higher than that of Thacker et al, particularly as they only studied patients with "convulsive" PNES; in our series, a history of events during sleep was mainly given by PNES patients with "convulsive" semiology. Our results suggest that a significant and greater than hitherto suspected proportion of patients with PNES may give a history of events during sleep at the time of clinical assessment. A history of events occurring exclusively during sleep may have some discriminative value between epileptic seizures and PNES, but a history of both sleep and waking events had no discriminative value at all in our sample.

At $58 \%$ overall, the proportion of PNES patients in our study who gave a history of events during sleep was surprisingly high. The doctor carrying out the interviews was experienced in the field, and care was taken to avoid leading questioning as far as possible. A major effect of leading questioning seems unlikely, given that patient accounts were confirmed by an eyewitness account of events arising during what appeared to be sleep in all but two cases. Care was also taken to be clear that clinical descriptions of sleep events were compatible with the patient's wake events and, at a later stage, that they were clinically compatible with events recorded during video EEG monitoring. We are therefore confident that our figure of $58 \%$ represents an accurate measurement in our sample.

The strong association we found between a history of events during sleep and social security benefits may be relevant. A purely mechanistic effect (that is, that patients giving a history of sleep events are simply more likely to be awarded benefit) would explain the association between the two, but would not explain the high prevalence of a history of events during sleep itself. However, epilepsy benefit forms in the United Kingdom have separate questions about nighttime events; this may have a suggestive effect in that the forms advise patients of the possibility of events during sleep and may suggest to patients (or their relatives) that having both daytime and night-time events indicates more severe disability. The associations we found between a history of events during sleep and simple measures of psychopathology (mood disorder, parasuicide) suggest that if this is so then the mechanism is unlikely to be simple malingering, and that factors other than benefits may also be at work. In any event, malingering is not considered a usual aetiological factor for PNES, though hard data are lacking, ${ }^{1}{ }^{10} 111^{15-17}$ This view is supported by a recent study of PNES, ${ }^{18}$ which showed that in a minority of patients, events began, if not straight from sleep, then almost immediately on arousal.

Not all studies agree that features commonly thought to indicate epilepsy rather than PNES are associated with a delay in diagnosis of PNES. ${ }^{19} 20$ We found no association between a history of events during sleep and a long time to diagnosis in patients with PNES. Our data do suggest that a history of events during sleep may encourage prescription of antiepileptic drugs, possibly because it reduces doctors' confidence in making a diagnosis of "no epilepsy," even if the diagnosis of PNES is already suspected.

We considered the possibility that a history of events during sleep in patients with PNES was simply a reflection of the severity or frequency of the PNES themselves, of the degree of associated disability, or of associated psychopathology. As this was part of a wider but more basic study, we chose a limited range of simple indices. We found a strong association with convulsive semiology, but no relation with event frequency when the data were analysed categorically. We did find associations with a history of parasuicide and mood disorder, which might suggest a relation with psychopathology that merits further investigation. In none of our patients who complained of fatigue was there a known medical explanation. Medically unexplained symptoms were common in our patients, at $51 \%$ overall, but there was no significant association with a history of events during sleep.

Benbadis et al found an association between pseudosleep PNES recorded on video EEG and a history of sexual abuse, though their numbers were small and the association did not reach statistical significance. ${ }^{10}$ Our own data also suggested a trend without reaching statistical significance: almost twice as many patients with a history of events during sleep admitted sexual abuse, with roughly similar figures for physical abuse (the coincidence of the two was common in our sample). Our data give no clear support for the hypothesis suggested by Fakhoury et al, ${ }^{21}$ that sleep PNES are associated with disturbance of sleep pattern, though again there was a trend. In a recent publication, ${ }^{22}$ a disturbance of sleep structure in patients with PNES was noted, but there was no comparison of patients with or without histories of events during sleep.

Recording PNES on video EEG usually allows a definitive diagnosis, and our data suggest that there should be greater readiness to use this technique at an earlier stage. During clinical assessment, certain features of the history may direct the clinician to consider the diagnosis of either PNES or epilepsy. Some features traditionally regarded as indicative of epilepsy-such as incontinence of urine and injuries (with the exception of burns)-do not in fact distinguish between epilepsy and PNES. ${ }^{53-26}$ A history from the patient or eyewitness of events during sleep may similarly misdirect the clinician, particularly a non-specialist, away from considering the diagnosis of PNES. It would perhaps be prudent to restate Gowers' "rule" as follows: "Pychogenic events do not arise from sleep, but may seem or be reported to do so."

\section{Authors' affiliations}

R Duncan, M Oto, A J C Russell, P Conway, West of Scotland Regional Epilepsy Service, Institute of Neurological Sciences, Southern General Hospital, Glasgow, UK

Competing interests: none declared

\section{REFERENCES}

1 Francis $\mathbf{P}$, Baker GA. Non-epileptic attack disorder (NEAD): a comprehensive review. Seizure 1999;8:53-61.

2 Sigurdardottir KR, Olafsson E. Incidence of psychogenic seizures in adults: a population based study in Iceland. Epilepsia 1998;39:749-52. 
3 Leis AA, Ross MA, Summers AK. Psychogenic seizures: ictal characteristics and diagnostic piffalls. Neurology 1992;42:95-9.

4 Lancman ME, Brotherton TA, Asconape JJ, et al. Psychogenic seizures in adults: a longitudinal analysis. Seizure 1993;2:281-6.

5 Meierkord $\mathrm{H}$, Will B, Fish D, et al. The clinical features and prognosis of pseudoseizures diagnosed using video-EEG telemetry. Neurology 1991;41:1643-6.

6 Gowers WR. Epilepsy and other chronic convulsive disorders. London: JA Churchill, 1901:328.

7 Desai BT, Porter RJ, Penry JK. Psychogenic seizures: a study of 42 attacks in 6 patients, with intensive monitoring. Arch Neurol 1982;39:202-9.

8 Lesser RP. Psychogenic seizures. In: Pedley TA, Meldrum BS, eds. Recent advances in epilepsy, vol 2. Edinburgh: Churchill Livingstone, 1985:273-96.

9 Manford M. Assessment and investigation of possible epileptic seizures. J Neurol Neurosurg Psychiatry 2001;70:113-18.

10 Benbadis SM, Lancman ME, King LM, et al. Preictal pseudosleep: a new finding in psychogenic seizures. Neurology 1996;47:63-7.

11 Thacker K, Devinsky O, Perrine K, et al. Non-epileptic seizures during apparent sleep. Ann Neurol 1993:33:414-18.

12 Lesser RP. Psychogenic seizures. Neurology 1996;46:1499-507.

13 Kanner AM, Morris HH, Luders $\mathrm{H}$, et al. Supplementary motor seizures mimicking pseudoseizures: some clinical differences. Neurology 1990:40:1404-7.

14 Saygi S, Katz A, Marks DA, et al. Frontal lobe partial seizures and psychogenic seizures: comparison of clinical characteristics. Neurology 1992;42:1274-7.
15 Bowman ES. Nonepileptic seizures: psychiatric framework, treatment, and outcome. Neurology 1999;53:S84-8.

16 Kuyk J, Leijten F, Meinardi $\mathrm{H}$, et al. The diagnosis of non-epileptic seizures: a review. Seizure 1997;6:243-53.

17 Betts TA, Boden S. Pseudoseizures (non-epileptic attack disorder). In: Trimble M, ed. Women and epilepsy. New York: John Wiley and Sons, 1991:243-58.

18 Orbach D, Ritaccio A, Devinsky O. Psychogenic nonepileptic seizures associated with video EEG verified sleep. Epilepsia 2003;44:64-8.

19 Reuber M, Fernandez G, Bauer J, et al. Diagnostic delay in psychogenic nonepileptic seizures. Neurology 2002;58:493-5

20 De Timary $\mathbf{P}$, Fouchet $\mathrm{P}$, Sylin $M$, et al. Non-epileptic seizures: delayed diagnosis in patients presenting with electroencephalographic and clinical signs of epileptic seizures. Seizure 2002;11:193-7.

21 Fakhoury T, Abou-Khalil B, Newman K. Psychogenic seizures in old age: a case report. Epilepsia 1993;34:1049-51.

22 Bazil CW, Legros B, Kenny E. Sleep structure in patients with psychogenic non epileptic seizures. Epilepsy Behav 2003;4:395-8

23 Peguero E, Abou-Khalil B, Fakhoury T, et al. Self injury and incontinence in psychogenic seizures. Epilepsia 1995;36:586-91.

24 Wilkus RJ, Dodrill CB, Thompson PM. Intensive EEG monitoring and psychological studies of patients with pseudoepileptic seizures. Epilepsia 1984;25:100-7.

25 Cohen RJ, Sluter G. Hysterical "seizures" - suggestion as a provocative EEG test. Ann Neurol 1982;11:391-5.

26 Gumnit R, Gates J. Psychogenic seizures. Epilepsia 1986;27(suppl):124-9. 